\title{
Investigation into the acceptability and effectiveness of a new contact slip in the management of Chlamydia trachomatis at a London genitourinary medicine clinic
}

\author{
A Wright, S Chippindale, D Mercey
}

See end of article for authors' affiliations

Correspondence to: Dr Danielle Mercey, Department of Sexually Transmitted Diseases, Royal Free and University College Medical School, University College London, UK; DMercey@gum.ucl.ac.uk

Accepted for publication 24 July 2002

\begin{abstract}
Objectives: To improve contact tracing for chlamydia. To determine (i) the acceptability to patients of using a contact slip that named chlamydia as the sexually transmissible infection that the contact may have been at risk of acquiring, and (ii) whether an augmented contact slip issued for chlamydia significantly increased the number of sexual partners attending for treatment.

Methods: For a 2 month period, all consecutive heterosexual patients diagnosed with chlamydia at the Mortimer Market Centre (MMC) were offered one or more coded contact slips (CS1) as per clinic protocol. During this time, and for 1 month afterwards, contacts presenting as a result of receiving these contact slips were recorded. This was compared to a subsequent 2 month period during which a contact slip naming chlamydia as the potential infection, with an information leaflet attached (CS2), was issued. Contacts attending as a result of receiving this augmented contact slip were recorded over a similar period. For both cohorts the number of patients refusing either contact slip, and contacts attending other GUM clinics, were recorded.

Results: 121 patients were diagnosed with chlamydia and were seen by a health adviser in period one and 130 patients were diagnosed and seen in period two. There was no significant difference detected between the acceptability of CS1 and CS2 (one refusal per cohort). There was a significant increase in the number of sexual contacts attending for treatment after being given CS2 (160 attending of 190 contact slips issued (84\%, 95\% Cl 79 to 89)), compared to CS1 (48 attending of 144 contact slips issued $(33 \%, 95 \% \mathrm{Cl} 26$ to 43$))$.

Conclusions: An infection specific contact slip was equally acceptable to patients as the standard contact slip, and it significantly increased the number of sexual contacts attending for treatment.
\end{abstract}

$\mathrm{P}$ artner notification has a key public health role in genitourinary medicine (GUM). The aim is for partners of index patients with a known sexually transmitted infection (STI) to attend for screening and treatment and therefore reduce the spread of infection and reinfection of untreated STIs. Contact slips are issued for each traceable contact identified, each slip (CS1) containing the index patient's clinic number, a coded diagnosis of the index patient's condition and the details of the issuing GUM clinic. The contact then presents the contact slip on attendance at a GUM clinic and receives the appropriate screening and medication. Details of the contact should then be cross referenced with the index case in order to establish whether partner notification has occurred. If the contact attends a clinic other than the one that issued the contact slip, the contact slip should be returned to the issuing clinic so that this cross referencing may occur. Despite the long established use of contact slips for patient referral and calls for their systematic evaluation ${ }^{1}$ few evaluations have been published.

In London the use of contact slips is problematic as there are many clinics a contact may attend. One audit revealed that of 80 contact slips issued, only 38 could be accounted for. ${ }^{2}$ Coded contact slips are intended to encourage index patients to bring their partners in for screening and/or treatment without necessarily disclosing their own diagnosis of an STI. However, it may be that the paucity of information deters the index patient from passing it to their partners and/or deters the contact from seeking screening and treatment.

Between 1998 and 1999 in England reports from the GUM clinics suggest at least a $20 \%$ increase in chlamydia. ${ }^{3}$ Effective partner notification for chlamydia is important because it often presents no symptoms in men or women unless it leads to complications. The only way a sexual contact may become aware of having chlamydia is through being informed by the index patient.

This study aimed to pilot and evaluate the use of a newly designed contact slip which incorporated the information on the traditional contact slip with an attached leaflet about Chlamydia trachomatis, including details of symptoms (or their absence), potential long term sequelae, information about attending a GUM clinic, and reassurance with regard to confidentiality (CS2).

Ethical approval for this study was obtained from the Camden and Islington Community (NHS) trust ethics committee.

The objectives of the study were to determine (i) the acceptability to patients of using a contact slip that named chlamydia as the STI that the contact may have been at risk of acquiring, and (ii) whether an augmented contact slip (CS2) issued for chlamydia significantly increased the number of sexual partners attending for treatment.

\section{METHOD}

Patients were eligible for this study if they were heterosexual, over 16 years of age, diagnosed with chlamydia, and their sexual partner(s) resided in the United Kingdom. All patients, who met the eligibility criteria of this study during October and November 2000 were issued with a CSI on referral to the health adviser, as per clinic protocol. For 3 months, starting in October, the number of contact attendances at Mortimer Market Centre (MMC) or elsewhere for each index patient was recorded using a standardised data collection sheet.

During February and March 2001 all eligible patients diagnosed with chlamydia who were referred to the health adviser 
Table 1 Comparison of contact slips issued and contact attendances

\begin{tabular}{llll}
\hline & $\begin{array}{l}\text { October- } \\
\text { November 2000 }\end{array}$ & $\begin{array}{l}\text { February-March } \\
2001\end{array}$ & Significance \\
\hline Total number of index patients & 210 & 214 & \\
Number of patients meeting inclusion criteria & 136 & 133 & \\
Number of patients not issued contact slip(s) & $33(24 \%)$ & $4(3 \%)$ & $p<0.0005$ \\
Number of patients issued contact slip(s) & $103(76 \%)$ & $129(97 \%)$ & $\left(\chi^{2}\right.$ test) \\
Number of contact slips issued & 144 & 190 & \\
Mean number of contact slips issued per patient & 1.4 (range 0-4) & 1.4 (range 0-4) & \\
Number of contacts attending for treatment & 48 & 160 & $p<0.0005$ \\
Contacts attending per contact slip issued & 0.3 & 0.8 & $\left(\chi^{2}\right.$ test) \\
Contacts attending per index patient & 0.5 (range 0-3) & 1.2 (range 0-4) & \\
\hline
\end{tabular}

Table 2 Reasons for non-issuing of contact slips

\begin{tabular}{lcl}
\hline & \multicolumn{2}{l}{ Number of patients } \\
\cline { 2 - 3 } Documented reason & $\begin{array}{l}\text { Oct-Nov } \\
2000\end{array}$ & $\begin{array}{l}\text { Feb-Mar } \\
2001\end{array}$ \\
\hline Did not attend for follow up & 1 & 1 \\
Patient not referred to health adviser & 11 & 2 \\
Did not wait for health adviser & 3 & 0 \\
Refused contact slip & 1 & 1 \\
Unable to contact partner & 17 & 0 \\
Total & 33 & 4 \\
\hline
\end{tabular}

as per clinic protocol, were offered CS2. For 3 months, starting in February, the number of contact attendances at MMC or elsewhere for each index patient was recorded.

For both cohorts, as well as recording the number of attendances verified as attending at both MMC and other GUM clinics, patients reporting partner attendance at other GUM clinics without the details required for verification (no surname, no date of birth, partner using a pseudonym, etc) were also recorded. Refusal of either contact slip was documented. Provision of care was otherwise identical, regardless of which contact slip, if any, was issued.

It was determined that in order to detect a $20 \%$ increase in number of contacts treated, with $90 \%$ power, each cohort needed to comprise at least 110 patients. The $\chi^{2}$ test was used to compare proportions between the two time periods.

\section{RESULTS}

In all, 136 and 133 patients, respectively, were eligible during each study period (table 1). A significantly higher proportion of patients was not given contact slips during the first period. The reasons are listed in table 2.

The number of refusals was similar in both cohorts (one in each).

The mean number of contact slips issued per patient was not different between the two groups (table 1).

The number of contacts attending per contact slip issued, and per index patient, was significantly higher with CS2 (table 1).

The proportion of contacts confirmed as, or merely reported to have been, treated elsewhere was similar in both groups (table 3).

\section{DISCUSSION}

The aim of partner notification is to break the chain of transmission of STIs and reduce the national rates of infections through identifying, counselling, and screening sexual partners/contacts of index patients and offering treatment if

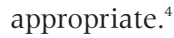

The CSI was designed to achieve this aim, as well as preserve the confidential diagnosis of the index patient through the use of a code in place of the name of the STI with which the index case has been diagnosed. In addition, use of CSI provides an acceptable means of identifying the potential infection to the clinic attended by the contact.

The first aim of this study was to determine whether identifying the infection on the contact slip would be less acceptable to the index patient. In the case of chlamydia infections in heterosexual patients, a contact slip naming the infection appears to be equally acceptable.

CS2 was significantly more effective as a means of encouraging sexual contacts to attend for screening and treatment. CSl achieved only a $45 \%$ rate of one or more contact attendance per index patient, less than the $70 \%$ minimum standard as documented in The Clinical Management of Genital Chlamydia Infection. ${ }^{5} \mathrm{CS} 2$, however, resulted in $89 \%$ rate of one or more contact attendance. CS2 also surpassed the standard of at least one contact per index patient attending, ${ }^{5}$ whereas CSI achieved only half of this target.

The study was not randomised, nor could it be blinded and there is, therefore, potential for bias. Staff may have been more enthusiastic about partner notification when using the new contact slip. There is some evidence for this as more patients were seen by a health adviser and therefore more patients received contact slips in the second study period. However, since more index patients were seen by health advisers in the second time period, it might be expected that this included more patients who were more reluctant to engage in partner notification. The number of contact slips issued per index patient seen remained the same, but the number of contacts attending per index patient increased, suggesting that the increase was due to the contact slip itself.

Health advisers may have more readily accepted that partner notification had been resolved in the second half of the study, but as every set of notes was reviewed by AW this was minimised. Furthermore, partner notification resolution was recorded on a specific proforma and there was no evidence of a higher proportion of "reported" as opposed to "confirmed" contacts treated, as shown in table 3.

Other clinics may have been more likely to return the new contact slip to the issuing clinic, leading to an apparently higher partner notification resolution rate for CS2. However, there is no evidence that a higher proportion of cases were treated elsewhere during the second part of the study (table 3).

Contacts from the CS2 cohort may have been encouraged to attend by the high profile health education awareness programmes in the media about chlamydia, and its potential long term sequelae, which began in 2000. Contacts, having been made aware of their potential risk of contracting chlamydia, may have found these programmes to be a catalyst in determining whether to attend for treatment.

Other studies have looked at ways of increasing the success of partner notification by using financial incentives or health 
Table 3 Comparison of partner notification resolution

\begin{tabular}{|c|c|c|c|c|c|}
\hline \multirow[b]{3}{*}{ Resolution definition } & \multicolumn{4}{|c|}{ Patients attending } & \multirow[b]{3}{*}{ Significance } \\
\hline & \multicolumn{2}{|c|}{ Oct-Nov 2000} & \multicolumn{2}{|c|}{ Feb-Mar 2001} & \\
\hline & Number & $\%$ & Number & $\%$ & \\
\hline \multicolumn{6}{|l|}{ Contact treatment } \\
\hline Confirmed at Mortimer Market Centre & 25 & 52 & 97 & 60 & \\
\hline Confirmed elsewhere & 3 & 6 & 14 & 9 & $p=0.35\left(\chi^{2}\right.$ test $)$ \\
\hline Reported & 20 & 42 & 49 & 31 & \\
\hline Total & 48 & $100 \%$ & 160 & $100 \%$ & \\
\hline
\end{tabular}

\section{Key messages}

- Contact tracing using contact slips is central to the public health role of STD clinics in England and Wales

- Despite this, there are no published reports of evaluations of variations on the standard contact slip

- This non-randomised study appears to show that a contact slip giving information about chlamydia infection is as acceptable and possibly more effective in contact tracing

education materials ${ }^{6}$ and by pre-booking contacts' appointments. $^{7}$ Only verbally delivered health education appeared to be effective in randomised controlled trials. Several of these studies used contact slips (or referral cards) but none has examined the content of these slips. Most studies of contact tracing have used "numbers of contacts traced" as the outcome measure, as we did in this study. It has been suggested that numbers of infected contacts is a better measure ${ }^{6}$ but van de Laar $e^{2} a^{8}$ have shown high rates of infection among traced contacts.

Further study of the use of such contact slips in other settings will provide information about the repeatability and generalisability of these findings. Studies focusing on other infections and other population groups will also be required.

\section{ACKNOWLEDGEMENTS}

This study was funded by a grant from the Clinical Research and Development Committee of the Royal Free and University College London Medical School.

\section{CONTRIBUTORS}

AW collated all the data, performed some of the analyses, wrote the first draft of the report, and reviewed all subsequent drafts; SC refined initial proposal, oversaw the data collection and work of AW, and reviewed all drafts of the report; DM conceived initial proposal, was research project supervisor, and reviewed all drafts of report.

\section{Authors' affiliations}

A Wright, S Chippindale, Mortimer Market Centre, off Capper Street, London WCIE 6AU, UK

D Mercey, Department of Sexually Transmitted Diseases, Royal Free and University College Medical School, University College London, UK

\section{REFERENCES}

1 Radcliffe K, Clarke J. Contact tracing - where do we go from here? Sex Transm Infect 1998:74:313-14.

2 Chippindale S, Robinson A. Relaunching chlamydia partner notification. Poster Presentation at MSSVD Spring Meeting. Athens 1998.

3 Robinson A. Chlamydia-the silent epidemic. www.netdoctor.co.uk/ womenshealth/facts/Chlamydia.htm 2001

4 SHASTD Working Group. Guidelines on partner notification. www.shastd.org.uk/professional_interest/partner.htm 1993

5 Central Audit Group in Genitourinary Medicine. The clinical management of genital chlamydia infection. www.shastd.org.uk/ professional-interest/chlamydi.htm 1997

6 Mathews C, Coetzee N, Zwarenstein M, et al. Strategies for partner notification for sexually transmitted diseases (Cochrane Review). In: The Cochrane Library, Issue I, 2002. Oxford: Update Software.

7 Patel HC, Viswalingam ND, Goh BT. Chlamydial ocular infection: efficacy of partner notification by patient referral. Int J STD AIDS 1994:5:244-7.

8 Van de Laar MJW, Termoshuizen F, van den Hoek A. Partner referral by patients with gonorrhea and chlamydial infection: case finding observations. Sex Transm Dis 1997:24:334-42. 